\title{
Generalized conditioned flavor aversions: Effects of toxicosis training with one flavor on the preference for different novel flavors
}

\author{
LINDA A. PARKER \\ University of New Brunswick, Saint John, New Brunswick, Canada \\ and \\ SAM REVUSKY \\ Memorial University of Newfoundland, St. John's, Newfoundland, Canada
}

\begin{abstract}
In two experiments, experimental rats were trained to have strong aversions to one of 10 novel-flavored solutions through contingent lithium injections; control rats were injected with lithium in the absence of prior consumption. Each animal was then tested with each of the 10 solutions, and the preferences of the experimental rats and the control rats were compared. A generalization gradient of the aversion to each conditioned aversive flavor was obtained for each test flavor.
\end{abstract}

Wild rats (Barnett, 1963; Richter, 1953; Rzoska, 1954), as well as laboratory rats (e.g., Best \& Batson, 1977; Carroll, Dinc, Levy, \& Smith, 1976; Domjan, 1976; Green \& Parker, 1975; Nachman \& Jones, 1974; Revusky \& Bedarf, 1967; Siegel, 1974), exhibit a tendency to avoid novel foods. This tendency, called neophobia, has been shown to be enhanced when rats have previously experienced illness following food consumption (Carroll et al., 1976; Domjan, 1975; Richter, 1953; Rozin, 1968; Revusky, Parker, Coombes, \& Coombes, 1976; Rzoska, 1954; Tapper \& Halpern, 1968), but not when rats have previously experienced illness in the absence of prior food consumption (Best \& Batson, 1977; Domjan, 1975; Revusky et al., 1976). Domjan (1975) has suggested that, in the former cases, an aversion to the flavor which preceded illness generalizes with the novel flavor, whereas in the latter cases, there is no opportunity for such stimulus generalization. When rats were injected with a toxin following consumption of saccharin, they drank less of a novel casein solution than did rats injected with a toxin in the absence of prior consumption (toxicosis alone), indicating that stimulus generalization occurred between saccharin and casein. There was no similar stimulus generalization between saccharin and vinegar.

The authors would like to thank Eric Holman for his valuable contributions to an earlier version of the manuscript and Shannon Coombes for technical assistance in conducting the experiments. Reprints may be obtained from Linda Parker, Division of Social Science, P.O. Box S050, University of New Brunswick, Saint John, New Brunswick, E2L 4L5, Canada.
Stimulus generalization has historically been explained in terms of common elements between stimuli. Thus, generalization between flavors may result from an overlap of common elements of a toxicosispaired flavor and a novel flavor and hence be evidenced as apparent enhanced neophobia after a poisoning experience. Psychophysical experiments have attempted to separate gustatory stimuli into four independent categories-sweet, sour, salty, and bitter; a "pure" flavor, containing elements exclusively from a single category, would not be expected to generalize with a flavor lacking elements of that category. Indeed, Nowlis and Frank (1977) demonstrated that generalization occurs between flavors which have been used in human psychophysical experiments to categorize the primary taste qualities. Furthermore, Nowlis, Frank, and Pfaffman (1980) established an aversion to one of 27 different flavored solutions in rats and hamsters and then tested them with each of four solutions prototypical of human's four taste qualities (sucrose, $\mathrm{NaCl}$, $\mathrm{HCl}$, and quinine). Each of the 27 flavors was shown to generalize with one of the four taste qualities in a manner predicted by human raters.

However, the flavors employed in flavor aversion learning experiments are typically not "pure"; for instance, saccharin (in high concentrations), a common CS, has both sweet and bitter components to human judges. The present study attempted to delineate a set of flavors that show minimal generalization with one another from a larger set of flavors commonly employed in experiments of flavor aversion learning. Such "orthogonal" flavors might be useful as stimuli in experiments that employ multiple flavor CSs. 
Since the designs of the following two experiments were complex, we will outline the logic of Experiment 1 here. Rats were divided into 10 training groups of nine rats each. These groups were trained, through contingent lithium injections, to have extremely strong aversions to solutions of (1) casein, (2) coffee, (3) grape juice, (4) milk, (5) quinine, (6) saccharin, (7) sucrose, (8) vinegar, or (9) $\mathrm{NaCl}$, or (10) they were subjected to toxicosis alone (that is, injected with lithium in the absence of prior consumption of a flavored solution). Each rat within these groups was subjected to a different testing sequence in which it consumed each of these nine flavored solutions once in a one-bottle test. The net result was that one rat from each training group drank casein on the first day, another rat drank it on the second day, and so on, in a balanced design. More specifically, the basic sequence was casein, coffee, sucrose, vinegar, $\mathrm{NaCl}$, milk, grape juice, quinine, saccharin. Variants of this sequence were derived by beginning with a flavor other than casein and continuing to the end of the basic sequence described above. For instance, if a rat began with a milk test, its sequence was milk, grape juice, quinine, saccharin, casein, coffee, sucrose, vinegar, $\mathrm{NaCl}$. Each of the nine rats in a training group began testing with a different one of the nine flavors. This procedure produced two measures of generalization between flavors $\mathbf{A}$ and $\mathbf{B}$. One was based on the preference for flavor $B$ among rats trained to have an aversion to flavor $\mathbf{A}$, and the other was based on the preference for flavor $A$ among rats trained to have an aversion to flavor $B$.

\section{METHOD}

\section{Experiment 1}

Subjects. Ninety male Sprague-Dawley rats, weighing 218-248 g, ad lib, were housed individually in stainless steel cages and removed only when weighed or injected.

Flavor solutions. Nine solutions were used: $5 \%(w / v)$ enzymatic casein hydrolysate, $1.25 \%$ (w/v) Sanka decaffeinated coffee, $50 \%$ (v/v) evaporated milk, $50 \%$ (v/v) Welch's grape juice, .01\% (w/v) quinine sulfate, $.4 \%(w / v)$ sodium saccharin, $20 \%(w / v)$ sucrose, $3 \%$ cider vinegar, and $1.5 \%(w / v)$ sodium chloride $(\mathrm{NaCl})$.

Conditioning trtals (Days 1, 2, and 5). Following 3 days of 10 min access to water in a bottle, the rats were conditioned. Nine rats were assigned, matched by body weight, to each of the 10 groups described in the introduction.

There were three conditioning trials (Days 1,2, and 5). On each trial, an experimental rat was presented with a bottle containing the appropriate flavored solution for $10 \mathrm{~min}$. As soon as the bottle was removed, the rat was injected intraperitoneally with $2 \% \mathrm{w} / \mathrm{v}$ $(.47 \mathrm{M})$ lithium chloride $(\mathrm{LiCl})$ in solution with distilled water; the doses on Days 1,2 , and 5 were 1,2 , and $3 \mathrm{ml}$, respectively. Nine control rats (toxicosis alone) were injected with the same volume of $\mathrm{LiCl}$ as the experimental rats, but did not drink earlier. Instead, they were presented with water for a 10 -min period which began $4 \mathrm{~h}$ after the $\mathrm{LiCl}$ injection; under such conditions, a learned aversion to unflavored water ought not to develop (e.g., Boland, 1973). On Conditioning Days 2 and 5, all rats were given $18 \mathrm{~h}$ of water from a bottle $1.5 \mathrm{~h}$ after the injection of $\mathrm{LiCl}$; there was no additional water presented on Conditioning Day 1. On a given trial, if a rat drank less than $3 \mathrm{ml}$, it received a $2-\mathrm{ml}$ infusion of the conditioning solution, washed across its tongue through a syringe in the course of about $10 \mathrm{sec}$, prior to the $\mathrm{LiCl}$ injection. After the final 18-h access to water, the rats were maintained on $12 \mathrm{~min}$ of access to water per day for 3 days (Days 7, 8, and 9).

Testing trials (Days 10-27). Nine test trials were given on alternate days over an 18-day period. On each trial, the rats received access to the appropriate test solution for $12 \mathrm{~min}$; on the intervening day, they received access to water for $12 \mathrm{~min}$. The sequence of test solution presentation was as described in the introduction.

On Test Day 1 , each rat from a given training $(\mathrm{CS}+)$ group drank a different test solution (TS); consequently, each test solution was consumed by 10 rats, one from each of the nine CS+ groups and one control. The same procedure was followed on each of the remaining 8 test days, according to the TS presentation sequence. By the completion of the 9 days, all rats had been exposed to each of the test solutions (including its own CS+ solution); however, one subject from each group began at a different point in the TS presentation sequence. Thus, for instance, there were to be nine tests of casein using rats made sick after drinking sucrose; one each of these tests was to be given on each of the days of testing. The TS preference scores of a given CS+ group were then pooled across test days. Of course, the rats would be expected to show stronger aversions in the earlier tests than in the later tests, because each test trial serves as an extinction trial when the CS+ flavor and the TS flavor share common elements. However, this effect of generalized extinction was minimized by establishing extremely strong aversions during conditioning, and any extinction effects were counterbalanced among training groups.

An unfortunate experimental error occurred on the first test day: Animals that were scheduled to receive $\mathrm{NaCl}$ were actually administered $1.5 \%$ sodium saccharin. Following the error, the sequence of test solution presentation was altered such that no other rats were tested with $\mathrm{NaCl}$. The data from the group in error were excluded from the data analysis. Thus, in Experiment 1, we have generalization scores between $\mathrm{NaCl}$ and other flavors only for animals trained on $\mathrm{NaCl}$ and tested on the other flavor; we have no data on the $\mathrm{NaCl}$ preferences of rats trained with other flavors.

\section{Experiment 2}

The procedures of Experiment 2 were identical to those of Experiment 1 except as indicated. The subjects were 110 male Sprague-Dawley rats weighing 215-240 g ad lib.

The groups differed from those of Experiment 1 in two ways: (1) To insure a relatively pure sour stimulus, a $1.5 \%$ normal $\mathrm{HCl}$ solution was added to the array of flavors. (2) The quinine solution concentration was reduced to $.005 \%$ to overcome a floor effect that was evident in the quinine intake of control rats in Experiment 1.

Each CS + group and the toxicosis-alone control group contained 10 rats. The conditioning trials and assignment to test conditions proceeded exactly as in Experiment 1, except for use of a different sequence of test-solution presentation: casein, milk, grape juice, quinine, $\mathrm{HCl}$, saccharin, $\mathrm{NaCl}$, coffee, sucrose, and vinegar. The testing procedure differed slightly. Rather than alternating test days and water days, 2 water days intervened between each test day to minimize differences in thirst levels between pairs of tests.

\section{RESULTS AND DISCUSSION}

Table 1 presents the mean amount consumed of each test solution (presented in rows) pooled across test days by rats in each CS condition (presented in columns). In each row, the upper number refers to Experiment 1 and the lower number refers to Experiment 2. The first column presents the mean amount consumed of each test flavor by the toxicosis-alone control group. 
Table 1

Mean Amount (in Milliliters) of Each Test Flavor (TS) Consumed by Each CS Group In Experiments 1 and 2

\begin{tabular}{|c|c|c|c|c|c|c|c|c|c|c|c|}
\hline \multirow{2}{*}{$\begin{array}{c}\text { Experi- } \\
\text { ment }\end{array}$} & \multicolumn{11}{|c|}{ CS Flavor } \\
\hline & Control & Casein & Coffee & Sugar & Vinegar & Milk & Grape Juice & Quinine & Saccharin & $\mathrm{NaCl}$ & $\mathrm{HCl}$ \\
\hline \multicolumn{12}{|c|}{ TS: Casein } \\
\hline 1 & 25.2 & $.9 \dagger$ & 21.9 & $13.4 *$ & $20.0^{*}$ & $8.1 *$ & $12.6^{*}$ & 20.1 & $3.7^{*}$ & $17.8^{*}$ & \\
\hline 2 & 16.5 & $1.2 \dagger$ & 11.2 & $7.3^{*}$ & $9.5^{*}$ & $2.5^{*}$ & $11.9^{*}$ & 13.0 & $2.5^{*}$ & $11.1 *$ & 12.8 \\
\hline \multicolumn{12}{|c|}{ TS: Coffee } \\
\hline 1 & 13.3 & 11.6 & $1.8 \dagger$ & 13.1 & $6.3 *$ & 11.8 & 10.2 & $7.1^{*}$ & 14.2 & 12.5 & \\
\hline 2 & 11.6 & 11.2 & $1.2 \dagger$ & 12.4 & $6.5^{*}$ & 11.5 & 9.3 & $8.2^{*}$ & 11.7 & 13.7 & 7.6 \\
\hline \multicolumn{12}{|c|}{ TS: Sugar } \\
\hline 1 & 21.8 & 15.3 & 18.1 & $1.2 \dagger$ & 20.1 & 16.1 & $12.9^{*}$ & 19.2 & $9.4^{*}$ & 15.8 & \\
\hline 2 & 17.5 & 12.7 & 18.9 & $.8 \dagger$ & 14.0 & 12.7 & $8.5^{*}$ & 16.1 & $3.4^{*}$ & 13.2 & 14.0 \\
\hline \multicolumn{12}{|c|}{ TS: Vinegar } \\
\hline 1 & 10.2 & 9.8 & $5.1^{*}$ & 11.9 & $.8 \dagger$ & 10.8 & $3.0^{*}$ & 8.0 & 10.6 & 9.3 & \\
\hline 2 & 9.7 & 8.2 & $7.4^{*}$ & 11.0 & $1.0 \dagger$ & 10.0 & $4.1^{*}$ & 6.1 & 8.3 & 8.5 & $3.1^{*}$ \\
\hline \multicolumn{12}{|c|}{ TS: Milk } \\
\hline 1 & 14.3 & $2.8^{*}$ & 10.4 & 10.2 & 12.1 & $.7 \dagger$ & $5.1^{*}$ & 13.3 & $7.3^{*}$ & 10.5 & \\
\hline 2 & 10.1 & $3.6^{*}$ & 8.3 & 7.8 & 8.0 & $1.7 \dagger$ & $8.4^{*}$ & 9.3 & $7.2^{*}$ & 8.2 & $7.7 *$ \\
\hline \multicolumn{12}{|c|}{ TS: Grape Juice } \\
\hline 1 & 10.2 & 7.4 & 7.2 & 5.4 & $1.6^{*}$ & $4.0 *$ & $.8 \dagger$ & 8.4 & 7.7 & 7.9 & \\
\hline 2 & 8.9 & 5.0 & 6.9 & 6.6 & $1.8^{*}$ & $7.0^{*}$ & $2.1 \dagger$ & 7.5 & 6.3 & 9.4 & $4.1^{*}$ \\
\hline \multicolumn{12}{|c|}{ TS: Quinine } \\
\hline 1 & 4.5 & 3.1 & $1.5^{*}$ & 6.3 & 2.9 & 7.4 & 3.5 & $.9 \dagger$ & 3.4 & 4.0 & \\
\hline 2 & 9.2 & 10.5 & $4.7^{*}$ & 9.5 & 7.4 & 9.1 & 10.0 & $2.1 \dagger$ & 6.0 & 5.5 & 6.2 \\
\hline \multicolumn{12}{|c|}{ TS: Saccharin } \\
\hline 1 & 15.1 & $1.8^{*}$ & 11.6 & $3.8^{*}$ & 11.6 & $6.2^{*}$ & $7.1 *$ & $8.4^{*}$ & $1.0 \dagger$ & $9.9^{*}$ & \\
\hline 2 & 14.1 & $4.2^{*}$ & 12.1 & $5.4^{*}$ & 10.4 & $6.9^{*}$ & $9.1^{*}$ & $6.7^{*}$ & $1.1 \dagger$ & $4.0^{*}$ & $9.6^{*}$ \\
\hline \multicolumn{12}{|c|}{ TS: $\mathrm{NaCl}$} \\
\hline 2 & 19.0 & 15.3 & 16.6 & 12.5 & 15.3 & 16.1 & 16.5 & 13.4 & 11.9 & $1.2 \dagger$ & 13.4 \\
\hline \multicolumn{12}{|c|}{ TS: $\mathrm{HCl}$} \\
\hline 2 & 12.6 & 11.1 & 9.9 & 13.0 & $4.2^{*}$ & 12.4 & $6.9^{*}$ & 8.8 & 10.8 & 9.9 & $2.3 \dagger$ \\
\hline
\end{tabular}

*CS flavor groups (pooled across Experiments 1 and 2) that significantly differed from the toxicosis-alone control group in amount of TS-flavored solution consumed $(p<.05)$. Groups that were tested with their CS+flavor were not included in the analysis.

fCS flavor groups that consumed the same test flavor with which they were conditioned.

In attempting to delineate a set of orthogonal flavor pairs from the array of flavored solutions, the amount consumed of each test-flavor solution (TS) was analyzed in a $2 \times 9$ unweighted-means ANOVA with the factors of experiment ( 1 or 2$)$ and CS condition. The group that was tested with its own CS+ flavored solution was excluded from the analysis. The $\mathrm{HCl} \mathrm{CS}+$ condition in Experiment 2 was not included in the overall analysis in order to maintain a balanced design. Instead, the $\mathrm{HCl} \mathrm{CS}+$ group was compared by $t$ tests with the control group for each TS in Experiment 2. Finally, the amounts consumed of $\mathrm{NaCl}$ and $\mathrm{HCl}$ TS in Experiment 2 (see Table 1) were analyzed as a single-factor ANOVA for CS condition.

In each TS flavor analysis, the experiment $\times$ CS condition interaction was not statistically significant. However, the factor of experiment was significant in the casein, sugar, milk, and quinine TS analyses. The rats that were tested with casein $\operatorname{TS}[F(1,153)=38.9$, $\mathrm{p}<.01]$, sugar $\mathrm{TS}[\mathrm{F}(1,153)=12.7, \mathrm{p}<.01]$, and milk TS $[F(1,153)=5.5, p<.01]$ drank more overall in Experiment 1 than in Experiment 2. In Experiment 1, the test days were separated by a single water day; in Experiment 2, the test days were separated by 2 water days, which may have allowed the rats to better satisfy their thirst and thus drink less of the novel test solutions on the test days. In contrast, rats drank less quinine TS in Experiment 1 than in Experiment 2 $[F(1,153)=40.0, p<.01]$, which was probably the result of the higher concentration of quinine solution used in Experiment 1.

The CS condition factor was significant in all TS analyses ( $p s<.05)$. The comparison of primary interest among the CS conditions for each TS was that between the toxicosis-alone control group and each of the remaining CS groups. These groups received equal experience with lithium and differed only on the basis of what they drank prior to the injection. Since the toxicosis-alone control group did not drink before being injected with lithium, they had no opportunity to generalize a flavor aversion to the TS 
flavor on the test days. Thus, if a group conditioned with a given CS flavor differed in TS intake from the toxicosis-alone controls, then that CS flavor showed some generalization with the TS flavor. In each row of Table 1, those CS groups that differed significantly by Newman-Keuls tests (ps $<.05$ ) from the toxicosis-alone controls (pooled across experiments) are represented by a single asterisk (*). Thus, these flavors showed some generalization with the TS flavor. The CS flavors with no asterisks did not differ from controls and may, thus, be considered relatively independent of the TS flavor. Although there are some instances in which a CS group had a mean consumption that was greater than the LiClalone controls, there was no case in which this difference was statistically significant. The mean amounts of TS flavors consumed by rats which were conditioned with the same CS flavors are represented diagonally in Table 1 with a dagger ( $\dagger$ ). In all cases, rats demonstrated greater avoidance of their CS flavor than did the toxicosis-alone controls $(p<.01)$.

For any TS analysis, when a CS flavor did not statistically differ from the toxicosis-alone controls, the CS flavor and the TS flavor were considered relatively independently of one another. However, there were actually two measures of generalization between any two flavored solutions, $A$ and $B$. The intake of solution $A$ among rats that had been trained to avoid solution $B$ and the intake of solution $B$ among rats that had been trained to avoid solution $\mathbf{A}$. For instance, in the analysis of the casein-coffee generalization, one measure is the intake of casein by the coffee CS group and the other measure is the intake of coffee by the casein CS group. As is evident in Table 1, by both measures, casein and coffee do not generalize with one another (as indicated by the absence of an asterisk). In order to describe any two flavors as independent, we require that such symmetrical independence occur between flavor pairs. The flavor pairs, from among the symmetrically independent data presented in Table 1, are listed in Table 2.

Symmetrical stimulus generalization occurred between the following flavor pairs: casein-saccharin,

Table 2

Set of Independent Flavor Pairs in Experiments 1 and 2

$\begin{array}{ll}\text { Casein-Coffee } & \text { Sugar-HCl } \\ \text { Casein-Quinine } & \text { Vinegar-Milk } \\ \text { Casein-HCl } & \text { Vinegar-Quinine } \\ \text { Coffee-Sugar } & \text { Vinegar-Saccharin } \\ \text { Coffee-Milk } & \text { Vinegar-NaCl } \\ \text { Coffee-Grape Juice } & \text { Milk-Quinine } \\ \text { Coffee-Saccharin } & \text { Milk-NaCl } \\ \text { Coffee-NaCl } & \text { Grape Juice-Quinine } \\ \text { Coffee-HCl } & \text { Grape Juice-NaCl } \\ \text { Sugar-Vinegar } & \text { Quinine-NaCl } \\ \text { Sugar-Milk } & \text { Quinine-HCl } \\ \text { Sugar-Quinine } & \text { NaCl-HCl }\end{array}$

Sugar- $\mathrm{NaCl}$

casein-milk, coffee-vinegar, coffee-quinine, sugarsaccharin, vinegar-grape juice, vinegar- $\mathrm{HCl}$, milkcasein, milk-grape juice, milk-saccharin, and grape juice- $\mathrm{HCl}$. Since these flavors were shown to generalize with one another regardless of which was the TS flavor and which was the CS+ flavor, they should not be used jointly as stimuli in experiments that employ multiple CS flavors.

There were also instances of asymmetrical generalization between flavor pairs. When either casein or saccharin served as the TS flavor, multiple instances (four each) of asymmetrical generalization were apparent. For instance, rats drank less saccharin TS than did the toxicosis-alone controls when they had been previously conditioned with grape juice; however, rats did not drink less grape juice TS than the controls when they had been previously conditioned with saccharin. With only two other TS flavors (sugar and milk) was there any further incidence of asymmetrical generalization, and in both cases there was only one such incident. It appears that casein and saccharin are unique in their tendency to generalize with other flavors. In fact, a post hoc $t$ test revealed that the difference in the number of instances of flavor generalization between saccharin/casein and all other TS flavors was significant $[t(8)=21.5$, $p<.001]$. The flavors of saccharin and casein may be less "pure" than the other flavors used in the present experiment and thus share a greater number of common stimulus elements with other CS+ flavors. Therefore, during the tests, the rats may detect elements which are common to the CS flavor and suppress their consumption of the TS solution. Interestingly, asymmetrical generalization has also been reported in more traditional paradigms (e.g., Moore, 1972).

The next question that was statistically analyzed was whether there were any instances in which the generalized aversion to a test flavor was as strong as the aversion to the CS+ flavor itself; we call such cases "complete generalization" between the two flavors. In all comparisons (pooled across Experiments 1 and 2), there were only two cases of complete generalization between flavors. Group Vinegar CS+ did not differ from Group Grape Juice CS+ when both groups drank grape juice TS $[t(36)=.78]$, nor did Group Vinegar CS+ differ from Group $\mathrm{HCl}$ $\mathrm{CS}+$ when both groups drank $\mathrm{HCl} \mathrm{TS}[\mathrm{t}(18)=1.60]$. However, neither effect was symmetrical, because when rats drank vinegar TS, Group Vinegar CS + significantly differed from both Group Grape Juice CS+ $[\mathrm{t}(36)=3.55, \mathrm{p}<.01]$ and Group $\mathrm{HCl} \mathrm{CS}+[\mathrm{t}(18)=$ $1.98, \mathrm{p}<.05]$. Since the complete generalization between the flavors was not symmetrical, the results must be interpreted cautiously.

Finally, the question of generalization of extinction was addressed. Although the sequence of flavor presentation was balanced among the subjects 
in each group, it is possible that exposure to the CS+ flavor during testing weakened the generalized aversions to the test solutions (TS). In such a case, TS intakes on days following a CS + trial should be greater than TS intakes on days preceding a CS+ trial. In order to evaluate this possibility, the test results are presented in Figure 1 as a function of test days. The upper graph presents the results of Experiment 1 , and the lower graph presents the results of Experiment 2. The upper set of data in each graph represents the amount consumed on each test day of all TS flavors other than the CS+ flavor. The closed circles represent the intakes by rats that had previously been tested with their CS + flavored solution, and the open circles represent the intakes by rats that had not yet been tested with their CS+ flavored solution. Unweighted means analyses of variance with prior $\mathrm{CS}+$ exposure and days as factors revealed that only the main effects of days was statistically significant, in both Experiment $1[\mathrm{~F}(5,436)=7.89, \mathrm{p}<.01]$ and Experiment $2[F(7,874)=14.6, p<.01]$. As is evident from the figure, rats drank less on earlier test days than on later test days. Since prior exposure to the CS+ flavor did not influence the intake of the test solutions, the increased intake across days was probably not the result of extinction of the flavor aversion to CS+.

Instead, the increased intake across days probably reflects adaptation to the 12 -min drinking schedule. This suggestion is supported by an analysis of the rats' 12-min water intake on the day prior to each test day in Experiment 2. The water intake data in Experiment 1 was not analyzed because only 1 day intervened between test days (in contrast to 2 days in Experiment 2), and thus the rat's water intake would be influenced by its intake of a given test solution on the preceding day. A single-factor ANOVA performed on the water-intake scores for all rats revealed a significant days effect $[F(9,1090)=30.3$, $\mathrm{p}<.001]$. The rats drank less water on the day prior to the earlier test days than on the day prior to the later test days. Thus, a similar pattern of consumption was evident whether the rats drank unflavored water or a novel flavored solution, which probably reflects adaptation to the drinking schedule over trials.

The lower set of data in both graphs in Figure 1 represents the amount of the CS+ flavors consumed on each test day. An analysis of variance in Experiment 1 revealed that there was a difference across days $[F(7,56)=2.69, p<.05]$, and Newman-Keuls tests showed that the Day 3 intake was greater (ps < .05) than on any other day. In Experiment 2, however, the days effect was not statistically significant $[F(9,90)=1.95, p<.05]$. The lack of an extinction effect across test days in Experiment 2 suggests that the Day 3 extinction effect in Experiment 1 may be

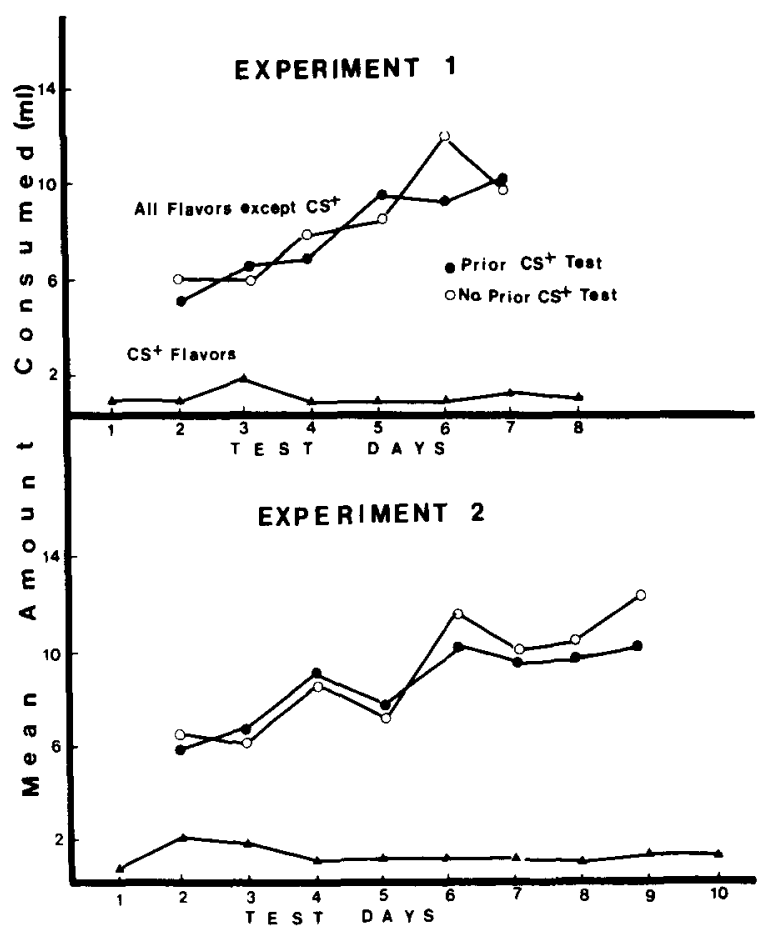

Figure 1. Mean consumption (pooled across navors) as a function of teat days. The upper set of datn points for each experiment represents the mean amount consumed of all favors, except the $\mathrm{CS}+$ flavor, on each test day. The solid circles $(\bullet)$ represent the nits which had previously received an extinction test trial with their $\mathrm{CS}+$ flavor, and the open circles $(O)$ represent the rats which had not yet recelved an extinction test trial with their $\mathrm{CS}+$ flavor. The triangles in the lower set of data points for each experiment (A) represent the mean amount of the pooled CS+ flavors consumed on each test day by rats that were tested with the same favor with which they were conditioned.

unreliable and that there was very little extinction of the aversions to the CS+ flavors as a result of prior exposure to other flavors.

Our results suggest that, as a consequence of prior toxicosis training with one flavored solution, some different novel flavors never associated with toxicosis are less likely to be consumed. Best and Batson (1977) have suggested that an aversion to a training flavor generalizes to a different test flavor because both flavors are novel to the animals; however, this "conditioned novelty aversion" cannot explain why some novel flavors do generalize and other novel flavors do not generalize with a conditioned aversive flavor.

As is evident in Table 1, each flavor had its own generalization gradient. Relatively orthogonal flavor pairs were obtained which correspond with the four primary taste categories. The following flavors showed no tendency to generalize with one another: sugar, $\mathrm{HCl}, \mathrm{NaCl}$, and either coffee or quinine (of course, these two bitter flavors did generalize with one another). Any other combination of four flavors showed some tendency to generalize; in fact, our 
results indicated that even sour vinegar generalized to some degree with bitter coffee (previously noted by Siegel, 1974).

Stimulus generalization has historically been explained in terms of common elements shared between stimuli. Our results show that, indeed, laboratory rats with previous experience with only the tastes inherent in rat chow and unflavored water were capable of discriminating between elements that were shared and elements that were different across a large number of flavored solutions. We suggest that investigators employing multiple CS experimental designs select their CS flavor pairs from those listed in Table 2.

\section{REFERENCES}

BaRnetr, S. A. The rat: A study in behavior. Chicago: Aldine Press, 1963.

Best, M. R., \& BAtson, J. D. Enhancing the expression of flavor neophobia: Some effects of the ingestion-illness contingency. Journal of Experimental Psychology: Animal Behavior Processes, $1977,3,132-143$.

Boland, F. J. Saccharin aversions induced by lithium chloride toxicosis in a backward conditioning paradigm. Animal Learning \& Behavior, 1973, 1, 3-4.

Brackbil., R. M., Rosenbush, S. M., \& Brookshire, K. H. Acquisition and retention of conditioned taste aversions as a function of the taste quality of the CS. Learning and Motivation, 1971, 2, 341-350.

Carroll, M. E., Dinc, H. I., Levy, C. J., \& Smith, J. C. Demonstrations of neophobia and enhanced neophobia in the albino rat. Journal of Comparative and Physiological Psychology, 1975, 89, 457.467.

DomJAN, M. Poison-induced neophobia in rats: Role of stimulus generalization of conditioned taste aversions. Animal Learning \& Behavior, 1975, 3, 205-211.
Domjan, M. Determinants of the enhancement of flavored-water intake by prior exposure. Journal of Experimental Psychology: Animal Behavior Processes, 1976, 2, 17-27.

Green, K. F., \& Parker, L. A. Gustatory memory: Incubation and interference. Behavioral Biology, 1975, 13, 359-367.

Moore, J. W. Stimulus control: Studies of auditory generalization in rabbits. In A. H. Black \& W. F. Prokasy (Eds.), Classical conditioning II: Current research and theory. New York: Appleton-Century-Crofts.

Nachman, M., \& Jones, D. R. Learned taste aversions over long delays in rats: The role of learned safety. Journal of Comparative and Physiological Psychology, 1974, 86, 949-956.

Nowlis, G., \& Frank, M. Qualities in hamster taste: Behavioral and neural evidence. Olfaction and Taste, 1977, 1, 241-248.

Nowlis, G., Frank, M., \& Prafrman, C. Specificity of acquired aversions to taste qualities in hamsters and rats. Journal of Comparative and Physiological Psychology, 1980, 94, 932-942.

Revusky, S., \& Bedarf, E. W. Association of illness with prior ingestion of novel foods. Science, 1967, 155, 219-220.

Revusky, S., Parker, L., Coombes, J., \& Coombes, S. Rat data which suggest that alcoholic beverages should be allowed during chemical aversion therapy, not just tasted. Behavior Research \& Therapy, 1976, 14, 189-194.

Richter, C. P. Experimentally produced behavior reactions to food poisoning in wild and domesticated rats. Annals of New York Academy of Sciences, 1953, 56, 225-239.

Rozis, P. Specific aversions and neophobia resulting from vitamin deficiency or poisoning in half-wild and domestic rats. Journal of Comparative and Physiological Psychology, 1968, 66, 82-88.

RzoskA, J. Bait shyness: A study in rat behavior. British Journal of Animal Behaviour, 1953, 1, 128-135.

SiEGEL, S. Flavor preexposure and "learned safety." Journal of Comparative and Physiological Psychology, 1974, 87, 1073-1082.

TAPPER, P., \& HALPERN, B. Taste stimuli: A behavioral categorization. Science, 1968, 161, 708-710.

(Manuscript received December 3, 1981; revision accepted for publication June 19, 1982.) 